\title{
The radiological diagnosis of bronchiectasis: what's in a name?
}

\author{
Harm A.W.M. Tiddens ${ }^{1,2}$, Jennifer J. Meerburg ${ }^{1,2}$, Menno M. van der Eerden ${ }^{3}$ \\ and Pierluigi Ciet $^{1,2}$
}

\author{
Number 3 in the Series "Controversies in bronchiectasis" \\ Edited by James Chalmers and Michal Shteinberg
}

Affiliations: ${ }^{1}$ Dept of Paediatric Pulmonology and Allergology, Erasmus Medical Centre (MC)-Sophia Children's Hospital, Rotterdam, The Netherlands. ${ }^{2}$ Dept of Radiology and Nuclear Medicine, Erasmus Medical Centre, Rotterdam, The Netherlands. ${ }^{3}$ Dept of Pulmonology, Erasmus Medical Centre, Rotterdam, The Netherlands.

Correspondence: H.A.W.M. Tiddens, Erasmus MC-Sophia Children's Hospital, Dept of Paediatric Pulmonology and Allergology, P.0 Box 2060, Room SP-3456, 3000 CB Rotterdam, The Netherlands.

E-mail: h.tiddensderasmusmc.nl

@ERSpublications

Bronchiectasis diagnosis is expert based. Clear definitions, standardisation of lung volume and CT protocols, and reference values are needed to allow automated image analysis for its diagnosis and to be used for clinical management and clinical studies. http://bit.ly/35vASqz

Cite this article as: Tiddens HAWM, Meerburg JJ, van der Eerden MM, et al. The radiological diagnosis of bronchiectasis: what's in a name? Eur Respir Rev 2020; 29: 190120 [https://doi.org/10.1183/16000617.0120-2019].

ABSTRACT Diagnosis of bronchiectasis is usually made using chest computed tomography (CT) scan, the current gold standard method. A bronchiectatic airway can show abnormal widening and thickening of its airway wall. In addition, it can show an irregular wall and lack of tapering, and/or can be visible in the periphery of the lung. Its diagnosis is still largely expert based. More recently, it has become clear that airway dimensions on CT and therefore the diagnosis of bronchiectasis are highly dependent on lung volume. Hence, control of lung volume is required during CT acquisition to standardise the evaluation of airways. Automated image analysis systems are in development for the objective analysis of airway dimensions and for the diagnosis of bronchiectasis. To use these systems, clear and objective definitions for the diagnosis of bronchiectasis are needed. Furthermore, the use of these systems requires standardisation of CT protocols and of lung volume during chest CT acquisition. In addition, sex- and age-specific reference values are needed for image analysis outcome parameters. This review focusses on today's issues relating to the radiological diagnosis of bronchiectasis using state-of-the-art CT imaging techniques.

Previous articles in this series: No. 1: Amati F, Simonetta E, Gramegna A, et al. The biology of pulmonary exacerbations in bronchiectasis. Eur Respir Rev 2019; 28: 190055. No. 2: Shteinberg M, Flume PA, Chalmers JD. Is bronchiectasis really a disease? Eur Respir Rev 2020; 29: 190051.

Provenance: Commissioned article, peer reviewed.

Received: 15 Sept 2019 | Accepted after revision: 02 Jan 2020

Copyright $\odot$ ERS 2020. This article is open access and distributed under the terms of the Creative Commons Attribution Non-Commercial Licence 4.0. 


\section{Introduction}

Bronchiectasis is derived from the Greek words bronckos meaning airway and ectasis meaning widening. However, depending on which specialist you are speaking to in the medical arena, bronchiectasis will have a different meaning and definition. A pathologist will associate bronchiectasis with the typical histological features, which include an abnormally widened airway with loss of the ciliary epithelial layer, a damaged epithelium and destruction of the elastin layer [1]. A radiologist will associate bronchiectasis with the typical chest computed tomography (CT) scan features, consisting of an abnormally widened and thickened airway with an irregular wall, lack of tapering and/or visibility of the airway in the periphery of the lung [2]. A (paediatric) pulmonologist will mostly diagnose bronchiectasis in a patient experiencing specific symptoms, such as dyspnoea, productive cough, loss of appetite and weight loss, recurrent airway infections and localised persistent rhonchi, with or without the radiological evidence of bronchiectasis $[3,4]$. In general, bronchiectasis is a man-made "diagnosis" that does not have the clear boundaries of diagnosis of conditions such as asthma or COPD. There are many causes of bronchiectasis, such as immune deficiencies, smoking-related airways disease, foreign body aspiration, recurrent gastric acid aspiration, cystic fibrosis (CF), autoimmune diseases, pneumonia and primary ciliary dyskinesia (PCD). However, in a large group of patients, no underlying disease was identified [5].

The diagnosis of bronchiectasis has varied over time. Before imaging, a diagnosis of bronchiectasis was primarily made post mortem through pathology findings. Imaging then allowed us to observe highly abnormally widened airways on chest radiographs, and later with more precision using bronchography techniques [6]. Since the mid-1990s, thanks to the development of high-resolution CT algorithms, chest CT became the gold standard for the diagnosis of bronchiectasis, as it was a more precise and less invasive technique than bronchography [2,7]. More recently, it was shown that it is possible to use magnetic resonance imaging (MRI) to diagnose bronchiectasis [8]. These more advanced diagnostic imaging techniques have allowed identification of the underlying pathophysiology of bronchiectasis in more patients. Hence, when reading literature on bronchiectasis, the timeframe should be taken into consideration. For this review, we will primarily focus on today's issues relating to the radiological diagnosis of bronchiectasis using state-of-the-art CT imaging techniques.

\section{Radiological definition of bronchiectasis}

In today's medical practice, the radiological diagnosis of $\mathrm{BE}$ is mostly made using chest $\mathrm{CT}$, as this technique is more sensitive than chest radiographs. However, the criteria for bronchiectasis are in general not well defined. When asking a radiologist about the definition of bronchiectasis, the answer will mostly include: "when the diameter of the airway is larger than the adjacent artery", "when an airway is clearly visible in the periphery of the lung", "when an airway has an irregular wall" and when "lack of tapering" can be observed. Interestingly, for these definitions, proper age- and sex-related reference values are lacking. These definitions probably work quite well in daily clinical practice for highly abnormally widened airways and/or in those who show a lack of tapering over multiple generations. For such advanced bronchiectasis airways, there will be fair to good inter- or intraobserver reproducibility of the diagnosis [9]. However, for the detection of early or mild stages of bronchiectasis disease, the radiological diagnosis becomes more critical and there will be more variability between observers. Precise and well-standardised diagnostic criteria are needed for the correct diagnosis of subtler airway changes.

When comparing the airway to artery (AA) ratio to diagnose bronchiectasis, a number of questions are unresolved. To begin with, should the inner or outer airway diameter be used for comparison with the adjacent artery? Currently, the inner airway diameter is generally used. However, this definition does not take into account the fact that the inner airway diameter of a widened airway can be reduced due to mucus attached to the airway wall and folding of the mucosa [10]. This results in a reduction of the internal diameter (figure 1) and also of the AA ratio. As a result, when using the internal diameter for comparison with the artery, such an airway might no longer meet the bronchiectasis criteria. In addition, in comparison with outer diameter, the inner diameter is more dependent on the lung volume level during CT acquisition [11].

At lung volumes below total lung capacity (TLC), the AA ratio will reduce more quickly when the inner airway diameter is used relative to the outer airway diameter [11, 12]. Hence, when the CT is performed at lung volumes below TLC, bronchiectasis may be missed when the inner airway diameter is used. Another issue with using the AA ratio for the diagnosis of bronchiectasis is the assumption that the arterial diameters remain unchanged in relation to lung disease. It is likely that there are conditions that can affect arterial diameters. The pulmonary artery system reduces blood flow to diseased areas of the lung thanks to the so-called hypoxic pulmonary vasoconstriction response. When this response includes a large region of the lung and when it persists for longer periods of time, it is possible that this will affect arterial diameters in such a region. Hence, the hypoxic pulmonary vasoconstriction response in diseased areas of the lung 

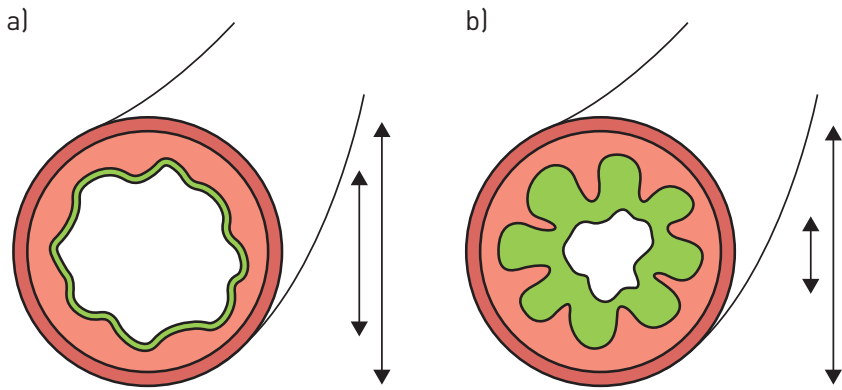

FIGURE 1 a) A healthy small airway and b) an inflamed airway with a thickened wall at full inspiration. On inspiration, the mucosa of the healthy airway is only slightly folded. The inflamed airway has larger folds compared with the normal airway. Mucus fills up the gaps between the mucosal folds. On chest CT, the folds and mucus will be interpreted as a thickened airway wall. This figure also illustrates why the outer diameter is a more robust parameter for diagnosing and quantifying bronchiectasis because in contrast to the inner diameter, it is not influenced by the presence of mucus in the lumen. Image provided by, and reproduced and modified with the kind permission of, M. Meerburg (Amsterdam, The Netherlands).

can result in an increase of the AA ratio. Other conditions that can reduce the dimensions of the pulmonary arteries are smoking and high altitude $[13,14]$. In chronic lung diseases, such as interstitial lung disease, the volume of pulmonary vessels tends to increase with the severity of disease [15].

Clearly, there is a need for age- and sex-related reference values for both airway and arterial dimensions. Such reference values should include children, because it is as yet unclear whether the same cut-off values for the AA ratio can be used as for adults and for children [11, 16-18]. To obtain such data is challenging as it will expose a healthy population to ionising radiation. Fortunately, the radiation doses needed for chest CTs have come down substantially, which has opened up the feasibility of using chest CTs in a healthy population. In addition, software is in development that allows the automatic and thus objective and reproducible measurements of AA dimensions of all visible AA pairs throughout the bronchial tree [19].

The presence of thickened and widened airways in the periphery of the lung is an important feature of bronchiectasis [20]. In the periphery of the lung, airways are small and are therefore mostly not visible, as their diameter is beyond the resolution of the CT scanner. It has been shown that in children with $\mathrm{CF}$, the number of airways visible on a chest CT scan was doubled in relation to control subjects [11]. This is largely due to small airways that have become visible thanks to thickening of the airway wall. On routine examination, these airways can easily be overlooked as being abnormal because their walls are thin.

For "lack of tapering", the other definition of bronchiectasis, objective and sensitive criteria are also lacking, similar to the AA ratio. Currently, lack of tapering is visually determined by the radiologist. Automated systems to assess inter- and intra-airway branch tapering are in development [21] and could be a sensitive approach to detecting airways disease. A major advantage of using tapering as a parameter of bronchiectasis, is that it does not require comparison to the pulmonary arteries, which makes it an important additional feature to the AA ratio. A challenge when analysing tapering is the automated system's recognition of airways that are occluded by mucus. Artificial intelligence techniques can be of help to reconstruct the complete bronchial tree to overcome this problem.

Therefore, for the objective and sensitive diagnosis of bronchiectasis on CT, objective quantitative criteria are needed to define widening, thickening and lack of tapering of the airways. To do so, age- and sex-dependent reference values for normal airway dimensions need to be developed.

\section{Standardisation of lung volume}

The importance of volume control during chest CT imaging has been understood since the late 1990s. Airway and arterial dimensions, parenchymal density and orientation of anatomical structures are all highly dependent on the respiratory phase and lung volume at which the chest CT is acquired (figure 2). Standardisation of the lung volume at TLC for the inspiratory CT scan and residual volume for an expiratory CT scan facilitates the objective and reproducible evaluation of AA dimensions, and reduces the risk of misinterpretation. Hence, volume standardisation for the acquisition of a chest CT fits the "as low as reasonably achievable" paradigm as the best diagnostic result will be obtained for the radiation given to the subject. Furthermore, standardising lung volume facilitates the longitudinal comparison between CT scans, as it allows slice by slice comparison.

Implementation of volume standardisation in cooperative patients is relatively easy to organise and has been extensively described $[22,23]$. Clinical implementation of this is not rocket science. In short, a 

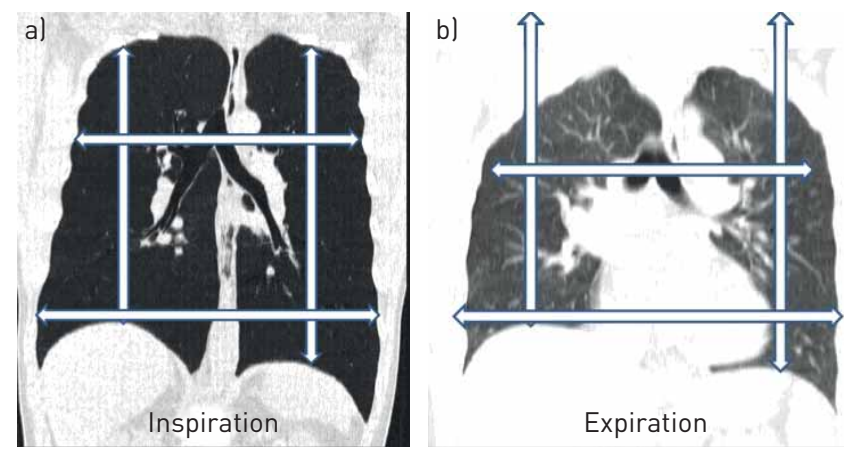

FIGURE 2 A spirometer-controlled chest computed tomography of a healthy adult showing the lung in the coronal plane at a) total lung capacity (TLC) and b) after full expiration at residual volume level. a) Arrows indicate the maximal height and width of the lung at TLC. b) The same dimensions projected over the lung in expiration. Note that the largest displacement is in the cranial caudal direction. Also note the differences in lung density and the altered orientation of the left and right main stem bronchi between inspiration and expiration. Finally, at full inspiration, the pleura parietalis is bulging out between the ribs, while on expiration the pleura parietalis is bulging in.

spirometry training session with the lung function scientist is scheduled on the day of the CT, generally half an hour prior to the CT scan. Lung function scientists are highly experienced in obtaining maximal respiratory effort from patients; they are practised in training patients and are able to coach patients to execute the correct breathing manoeuvres during CT scanning. The training session serves to: train the patient to execute the appropriate breathing manoeuvres; obtain the targeted lung volumes; collect spirometry values to be used during scanning; and alleviate anxiety by familiarising the patient with the scanner, the technique and instructions. Half an hour is generally sufficient for nearly all patients $\geqslant 5$ years of age to achieve the training goals. In our centre the session is scheduled just prior to the scan in the lung function department, or in a preparation room close to the scanner. During the scan, the lung function scientist coaches the patient and collaborates closely with the radiographer, who can focus on operating the scanner. Importantly, the spirometer-controlled procedure does not take up more time in the scanner for the patient; therefore, there is no reduction in CT productivity.

Spirometer-guided chest imaging is a viable method that can be implemented in cooperative patients. It was introduced in our centre for school-aged children as part of our clinical routine in 2007, and it has been successful in $90 \%$ of scans [22]. The technical equipment needed and the logistics required to implement spirometer-guided chest imaging are feasible for most centres. Where it is not feasible for a patient to use a spirometer, a good volume result can also be obtained when breathing manoeuvres are given by the lung function technician during the CT scan.

Therefore, volume standardisation of chest CTs can and should be implemented in clinical routine as a shared responsibility of pulmonologists and radiologists.

\section{Standardisation of CT protocols}

As for standardisation of the lung volume of chest CTs, a higher level of standardisation of CT scan settings and image reconstruction is required for the sensitive evaluation of airway dimensions. In today's radiology practice, chest CT protocols still vary widely between and even within hospitals, and often depend on local preferences by radiologists [24]. The following CT settings need to be standardised.

Volumetric techniques should be used. It is also important that a CT protocol is used that allows the reconstruction of $\leqslant 1 \mathrm{~mm}$ slices. Such a protocol is often referred to as a high-resolution CT. For evaluation, the images should be reconstructed with a slice thickness of $\leqslant 1 \mathrm{~mm}$ in the axial, sagittal and coronal plane, meaning airways can easily be followed through the bronchial tree, thereby making it possible to differentiate bronchiectasis from other abnormalities, such as cysts, abscesses and bulla. This CT protocol also allows a slice by slice comparison between two chest CT scans to evaluate disease progression. In addition, slice thickness is an important determinant of airway wall thickness. The wall of an airway cut in cross section appears thicker on a 3- or 5-mm reconstructed slice compared with a $1 \mathrm{~mm}$ slice.

Reconstruction kernels should be standardised, as they impact upon the appearance of the lung tissue. For the comparison of two consecutive CTs, reconstruction filters should be similar. To allow the use of automated analysis methods, it is recommended that a series that uses filtered back projection is included where available $[19,25]$. If using iterative reconstruction techniques, it is recommended that a kernel specifically designed for quantitative analysis is included. 
Finally, a low-dose CT protocol can be used as it been shown that low-dose thin-section CTs are sufficiently sensitive for the detection of bronchiectasis in paediatric and adult patients [26, 27].

To achieve uniformity in CT protocols within and between centres, task forces involving respiratory societies, radiology societies and image analysis companies are required. These standardisation efforts are a prerequisite for the inclusion of chest CT-related bronchiectasis outcome measures that use automated image analysis tools in registries and clinical trials [24].

\section{Image analysis}

Today's radiology reports of chest images for routine care are still largely expert based, mostly unstandardised and generally do not contain quantitative outcome data. In other clinical specialities like cardiology, quantitative post-processing methods to acquire objective measures of coronary artery stenosis and of pulmonary artery and aorta dimensions are available and well-implemented [28, 29].

For bronchiectasis patients, there are currently three multidimensional scores that include radiological assessment: the Bronchiectasis Severity Index (BSI) [30]; the FACED (forced expiratory volume in $1 \mathrm{~s}$ $\left(\mathrm{FEV}_{1}\right)$, age, chronic colonisation, extension and dyspnoea) score [31]; and the E-FACED (FACED plus exacerbations) score [32]. In the BSI, 1 point is assigned where the number of affected lobes is three or higher, or where cystic bronchiectasis is present (maximum BSI score: 26). Other parameters are age, body mass index, $\mathrm{FEV}_{1} \%$ predicted, hospital admissions, exacerbations, dyspnoea score, Pseudomonas aeruginosa colonisation and colonisation with other organisms. In the FACED score, 1 point is assigned where more than two lobes are affected (max score: 7 points). Other parameters are chronic P. aeruginosa colonisation, dyspnoea score, $\mathrm{FEV}_{1} \%$ pred and age. For the E-FACED score, the number of exacerbations in the year before the CT scan is included to predict future yearly exacerbations.

The Bronchiectasis Radiologically Indexed CT Score (BRICS) is a very quick and simple radiological score that was developed for use in bronchiectasis patients [33]. It contains the amount of bronchial dilatation and the number of bronchopulmonary segments with emphysema, resulting in an outcome between 0 and 5 . Due to their multi-dimensional character and simplicity, these scores are unlikely to pick up subtle changes on chest CTs to monitor disease progression and to be used as outcome measure in clinical trials.

More detailed semi-quantitative scoring systems have been developed to quantify bronchiectasis and other key characteristics related to CF [34-37]. Initially, these systems were developed for research purposes but increasingly they find their way into routine clinical care. More recently, a quantitative grid-based method (the Perth-Rotterdam Annotated Grid Morphometric Analysis for CF (PRAGMA-CF)) was developed based on morphometric principles [37]. It was shown to be sensitive for quantifying and monitoring airway changes, including bronchiectasis [11]. Bronchiectasis-related semi-quantitative and PRAGMA-CF scores have been shown to correlate well with more objective assessments of AA dimensions [38]. Quantitative grid-based methods are now also being developed and applied for PCD and for bronchiectasis studies (H.A.W.M. Tiddens; personal communication). An advantage of grid-based scoring systems is that it can be automated using machine learning techniques.

Another promising method for the detection of airways disease is the objective measurement of airway [25] or AA dimensions [11]. On a chest CT scan, hundreds of airways and AA pairs can be measured. Manual measurement of AA dimensions in CF has shown early and diffuse widening of the airways and airway wall thickening throughout the bronchial tree, with higher mean AA ratios and more severe thickening in the smaller airways [11]. An important observation was that in children with normal chest CTs, the mean outer AA ratio was 1.1 and was independent of segmental generation. However, outer AA ratios of $>1.1$ could also be detected on the chest CTs of control subjects without lung disease. There are reasons to believe that this ratio might also change in relation to age [17]. On the chest CTs of young subjects without lung disease, the bronchial tree can be followed up to the seventh segmental generation, depending on age and size of the subject. For diseases such as CF where the small airways are heavily involved, the bronchial tree can become visible all the way up to the 12th segmental generation [11]. In healthy adults, automated analyses can detect 10-14 segmental generations [39, 40]. Unfortunately, manual measurements of all AA pairs on a chest CT is hugely time consuming, and therefore not suitable for routine clinical care. Fortunately, image analysis techniques that allow automated analysis of all visible AA pairs are in development [19]. As previously discussed, age- and sex-specific reference values need to be developed that allow the comparison of single patient outcomes with reference values. As radiation doses have come down considerably for chest CT scans, collection of such data might become feasible. Clinicians can use these chest CT outcome measures for the objective assessment and monitoring of bronchiectasis patients, in a similar way to spirometry outcomes.

These automated systems will be of great value to registries such as the European Multicentre Bronchiectasis Audit and Research Collaboration (EMBARC) [41] and the European Cystic Fibrosis 
Society Patient Registry. Adequate phenotyping and quantification of structural lung abnormalities on chest CT using automated image analysis systems will improve our understanding of the pathophysiology of bronchiectasis and will be important to the improvement of patient care.

\section{Reversibility of bronchiectasis}

Bronchiectasis is generally defined as an irreversible widening of the airway. The reversibility of radiological bronchiectasis has been discussed in a number of case reports [42, 43], and in a few small retrospective studies including both adult and paediatric patients [44-47]. A few critical notes are needed. In some of these papers [43], the methodology of both image acquisition and analysis are not well defined. This is highly relevant, as a volumetric CT protocol is required to allow a slice by slice comparison at identical anatomical levels between the baseline scan and the follow-up scan. When sequential CT protocols are used, this is not usually possible and smaller bronchiectasis visible on the baseline scan can therefore be easily missed on the follow-up scan. This is especially relevant for older studies reporting reversibility of bronchiectasis when sequential protocols were more likely to be used [45, 47]. Differences in slice thickness and/or image reconstruction kernels between the baseline CT scan and follow-up scan can also result in "disappearance" of a bronchiectasis airway. Furthermore, reduction of bronchial wall thickening in the follow-up scan of smaller more peripheral airways after a therapeutic intervention can reduce visibility or make it disappear due to limitations in resolution. Sometimes airways with a normal diameter are misdiagnosed as bronchiectasis because of a thickened airway wall. Such thickening can be reversed in response to effective therapy. Differences in lung volume between baseline and follow-up CT can also result in the "disappearance" of bronchiectasis.

In general, advanced bronchiectasis is an irreversible condition. For critical follow-up of subtle bronchiectasis, the same standardised sensitive CT protocol needs to be used for both the baseline and follow-up scan.

\section{Differentiation between diffuse and local disease}

For the management of bronchiectasis, it is important to understand whether the condition developed as the result of an underlying chronic disease or whether it developed in relation to a single event, such as pneumonia of foreign body aspiration. In CF lung disease, bronchiectasis is the result of chronic and diffuse airway inflammation and infection. Measurements of all AA pairs visible on chest CT have shown that subtle but diffuse and progressive airway widening and thickening can be detected in patients as young as 2-4 years old [18]. With increasing age, more AA pairs show abnormal thickening and widening. For CF, it has therefore become clear that bronchiectasis is the "tip of the iceberg" and an end-stage local condition of progressive and diffuse airway disease. These and other related observations have led to a paradigm shift in the treatment of CF, namely that airway disease is diffuse and starts in infancy, and that it should be treated at an early stage to prevent the development of bronchiectasis [12]. Whether this phenomenon of mild diffuse airway disease preceding the development of bronchiectasis also occurs in other diseases, such as PCD, or immune deficiencies, needs to be further investigated. Clearly, when mild diffuse airway disease leads to bronchiectasis, early detection and treatment are also relevant for these diseases to prevent development into bronchiectasis.

In bronchiectasis patients without diffuse airway disease, the pathophysiology of bronchiectasis is more likely to be the result of a local event, such as pneumonia or whooping cough. Hence, we feel that distinguishing between diffuse or local airways disease can be important for the differential diagnosis of the underlying disease causing bronchiectasis, and for the management of such a disease. We speculate that a sensitive read-out of all visible AA ratios throughout the bronchial tree can be helpful for differentiating between diffuse or local disease.

\section{MRI and bronchiectasis}

Obtaining detailed and reproducible morphological images of the lung with MRI in a clinical routine that is comparable to that of CT is still a major challenge. However, lung imaging using MRI has made substantial progress over the last two decades [48]. As for CT, consensus on the classification of bronchiectasis is lacking. Furthermore, standardisation across centres and vendors for lung MRI is in its early days [49-51]. However, there have been important developments that may significantly contribute to the future of bronchiectasis research, as follows.

First, new sequences are in development that allow high-resolution lung images to be obtained [52, 53].

Secondly, MRI allows more detailed tissue characterisation in comparison with CT. Hence, when using MRI, the contribution of mucus to airway wall thickening can be investigated [54, 55]. Such techniques can be of great importance when studying, for example, physiotherapy techniques in bronchiectasis patients to improve mucocilliary clearance [56].

Thirdly, MRI techniques like diffusion weighted imaging are in development to identify inflammatory hotspots that can potentially be used to monitor treatment efficacy [57]. 
Fourthly, using functional MRI, the relation between perfusion and ventilation can be studied. A concept has been put forward to develop a set of sequences to obtain information on ventilation inflammation perfusion and structure (VIPS-MRI) in one session in CF patients [58]. The challenge is that the set of sequences: can be completed in $\sim 30 \mathrm{~min}$; does not require a contrast agent; and runs on all major MRI platforms. Clearly, the VIPS-MRI platform could be of interest for all lung diseases that include severe bronchiectasis.

MRI is therefore likely to play a more important role in the near future for bronchiectasis-related research. However, for larger scale use in the day-to-day clinical practice of bronchiectasis, important issues such as standardisation, spatial resolution, accessibility and validation of outcome measures need to be addressed.

\section{Summary}

The gold standard for bronchiectasis diagnosis in vivo over the last two decades has been chest CT. Bronchiectasis is diagnosed when an abnormally wide and mostly thickened airway can be observed, which often also shows a lack of tapering. Bronchiectasis can be the result of a clearly defined underlying disease, but in many patients, the underlying disorder that led to bronchiectasis cannot be identified. Standardisation of lung volume during chest CT acquisition and of the scanner protocol is needed to allow a more robust diagnosis of bronchiectasis. In addition, this standardisation is needed to allow automated sensitive image analysis for the objective diagnosis of bronchiectasis. It is important that outcome measures derived from automated image analysis methods of chest CTs are used in clinical care and registries. Chest MRI can play an important role in bronchiectasis research, allowing the study of both lung structure and functional aspects.

Conflict of interest: H.A.W.M. Tiddens has received other funding from Roche and Novartis for symposium, lecture fees and advisory boards. He has received grants from CFF, Vertex, Gilead, Chiesi and Vectura outside the submitted work. $\mathrm{He}$ also has a patent pending for the PRAGMA-CF scoring system. He is head of the Erasmus MC core laboratory Lung Analysis which is a not-for-profit core image analysis laboratory. The financial aspects of the laboratory are handled by the department of Radiology and by the Sophia Research BV. FLUIDDA has developed computational fluid dynamic modelling based on chest CTs obtained from Erasmus MC-Sophia for which royalties are received by Sophia Research BV. J.J. Meerburg has nothing to disclose. M.M. van der Eerden has nothing to disclose. P. Ciet has nothing to disclose.

\section{References}

1 Boaventura R, Shoemark A, Chalmers JD. Pathophysiology. In: Chalmers JD, Polverino E, Aliberti S, eds. Bronchiectasis (ERS Monograph). Sheffield, European Respiratory Society, 2018; pp. 8-28.

2 Hansell DM, Bankier AA, MacMahon H, et al. Fleischner Society: glossary of terms for thoracic imaging. Radiology 2008; 246: 697-722.

3 Polverino E, Goeminne PC, McDonnell MJ, et al. European Respiratory Society guidelines for the management of adult bronchiectasis. Eur Respir J 2017; 50: 1700629.

4 McDonnell MJ, Aliberti S, Goeminne PC, et al. Comorbidities and the risk of mortality in patients with bronchiectasis: an international multicentre cohort study. Lancet Respir Med 2016; 4: 969-979.

5 Chandrasekaran R, Mac Aogain M, Chalmers JD, et al. Geographic variation in the aetiology, epidemiology and microbiology of bronchiectasis. BMC Pulm Med 2018; 18: 83.

6 Hedblom CA. The diagnosis and treatment of bronchiectasis. JAMA 1927; 89: 1384-1390.

7 McGuinness G, Naidich DP. CT of airways disease and bronchiectasis. Radiol Clin North Am 2002; 40: 1-19.

8 Dournes G, Menut F, Macey J, et al. Lung morphology assessment of cystic fibrosis using MRI with ultra-short echo time at submillimeter spatial resolution. Eur Radiol 2016; 26: 3811-3820.

9 de Brito MC, Ota MK, Leitao Filho FS, et al. Radiologist agreement on the quantification of bronchiectasis by high-resolution computed tomography. Radiol Bras 2017; 50: 26-31.

10 Lambert RK, Codd SL, Alley MR, et al. Physical determinants of bronchial mucosal folding. J Appl Physiol 1994; 77: 1206-1216.

11 Kuo W, de Bruijne M, Petersen J, et al. Diagnosis of bronchiectasis and airway wall thickening in children with cystic fibrosis: objective airway-artery quantification. Eur Radiol 2017; 27: 4680-4689.

12 Mott LS, Graniel KG, Park J, et al. Assessment of early bronchiectasis in young children with cystic fibrosis is dependent on lung volume. Chest 2013; 144: 1193-1198.

13 Diaz AA, Young TP, Maselli DJ, et al. Quantitative CT measures of bronchiectasis in smokers. Chest 2017; 151: $1255-1262$.

14 Kim JS, Muller NL, Park CS, et al. Bronchoarterial ratio on thin section CT: comparison between high altitude and sea level. J Comput Assist Tomogr 1997; 21: 306-311.

15 Jacob J, Bartholmai BJ, Rajagopalan S, et al. Mortality prediction in idiopathic pulmonary fibrosis: evaluation of computer-based CT analysis with conventional severity measures. Eur Respir J 2017; 49: 1601011.

16 Matsuoka S, Uchiyama K, Shima $\mathrm{H}$, et al. Bronchoarterial ratio and bronchial wall thickness on high-resolution CT in asymptomatic subjects: correlation with age and smoking. AJR Am J Roentgenol 2003; 180: 513-518.

17 Copley SJ, Wells AU, Hawtin KE, et al. Lung morphology in the elderly: comparative CT study of subjects over 75 years old versus those under 55 years old. Radiology 2009; 251: 566-573.

18 Kuo W, Soffers T, Andrinopoulou E, et al. Quantitative assessment of airway dimensions in young children with cystic fibrosis lung disease using chest computed tomography. Pediatr Pulmonol 2017; 52: 1414-1423.

19 Perez-Rovira A, Kuo W, Petersen J, et al. Automatic airway-artery analysis on lung CT to quantify airway wall thickening and bronchiectasis. Med Phys 2016; 43: 5736. 
20 Naidich DP, McCauley DI, Khouri NF, et al. Computed tomography of bronchiectasis. J Comput Assist Tomogr 1982; 6: 437-444.

21 Kuo W, Perez-Rovira A, Tiddens $\mathrm{H}$, et al. Airway tapering: an objective image biomarker for bronchiectasis. Eur Radiol 2020; in press [doi: 1007/s00330-019-06606-w].

22 Salamon E, Lever S, Kuo W, et al. Spirometer guided chest imaging in children: it is worth the effort! Pediatr Pulmonol 2017; 52: 48-56.

23 Kongstad T, Buchvald FF, Green K, et al. Improved air trapping evaluation in chest computed tomography in children with cystic fibrosis using real-time spirometric monitoring and biofeedback. J Cyst Fibros 2013; 12 : 559-566.

24 Kuo W, Kemner-van de Corput MP, Perez-Rovira A, et al. Multicentre chest computed tomography standardisation in children and adolescents with cystic fibrosis: the way forward. Eur Respir J 2016; 47: 1706-1717.

25 Wielputz MO, Eichinger M, Weinheimer $\mathrm{O}$, et al. Automatic airway analysis on multidetector computed tomography in cystic fibrosis: correlation with pulmonary function testing. J Thorac Imaging 2013; 28: 104-113.

26 Kubo T, Ohno Y, Nishino M, et al. Low dose chest CT protocol (50 mAs) as a routine protocol for comprehensive assessment of intrathoracic abnormality. Eur J Radiol Open 2016; 3: 86-94.

27 O'Connor OJ, Vandeleur M, McGarrigle AM, et al. Development of low-dose protocols for thin-section CT assessment of cystic fibrosis in pediatric patients. Radiology 2010; 257: 820-829.

28 Johnson PT, Fishman EK. Postprocessing techniques for cardiac computed tomographic angiography. Radiol Clin North Am 2010; 48: 687-700.

29 Schulz-Menger J, Bluemke DA, Bremerich J, et al. Standardized image interpretation and post processing in cardiovascular magnetic resonance: Society for Cardiovascular Magnetic Resonance (SCMR) board of trustees task force on standardized post processing. J Cardiovasc Magn Reson 2013; 15: 35.

30 Chalmers JD, Goeminne P, Aliberti S, et al. The bronchiectasis severity index. An international derivation and validation study. Am J Respir Crit Care Med 2014; 189: 576-585.

31 Martinez-Garcia MA, de Gracia J, Vendrell Relat M, et al. Multidimensional approach to non-cystic fibrosis bronchiectasis: the FACED score. Eur Respir J 2014; 43: 1357-1367.

32 Martinez-Garcia MA, Athanazio RA, Giron R, et al. Predicting high risk of exacerbations in bronchiectasis: the E-FACED score. Int J Chron Obstruct Pulmon Dis 2017; 12: 275-284.

33 Bedi P, Chalmers JD, Goeminne PC, et al. The BRICS (Bronchiectasis Radiologically Indexed CT Score): a multicenter study score for use in idiopathic and postinfective bronchiectasis. Chest 2018; 153: 1177-1186.

34 Bhalla M, Turcios N, Aponte V, et al. Cystic fibrosis: scoring system with thin-section CT. Radiology 1991; 179: 783-788.

35 Brody AS, Klein JS, Molina PL, et al. High-resolution computed tomography in young patients with cystic fibrosis: distribution of abnormalities and correlation with pulmonary function tests. J Pediatr 2004; 145: 32-38.

36 Wainwright CE, Vidmar S, Armstrong DS, et al. Effect of bronchoalveolar lavage-directed therapy on Pseudomonas aeruginosa infection and structural lung injury in children with cystic fibrosis: a randomized trial. JAMA 2011; 306: 163-171.

37 Rosenow T, Oudraad MC, Murray CP, et al. PRAGMA-CF. A quantitative structural lung disease computed tomography outcome in young children with cystic fibrosis. Am J Respir Crit Care Med 2015; 191: 1158-1165.

38 Kuo W, Andrinopoulou ER, Perez-Rovira A, et al. Objective airway artery dimensions compared to CT scoring methods assessing structural cystic fibrosis lung disease. J Cyst Fibros 2017; 16: 116-123.

39 Montaudon M, Desbarats P, Berger P, et al. Assessment of bronchial wall thickness and lumen diameter in human adults using multi-detector computed tomography: comparison with theoretical models. J Anat 2007; 211: 579-588.

40 Kirby M, Tanabe N, Tan WC, et al. Total airway count on computed tomography and the risk of chronic obstructive pulmonary disease progression. Findings from a population-based study. Am J Respir Crit Care Med 2018; 197: 56-65.

41 Chalmers JD, Aliberti S, Polverino E, et al. The EMBARC European Bronchiectasis Registry: protocol for an international observational study. ERJ Open Res 2016; 2: 00081-2015.

42 Hayes D Jr, Long FR, McCoy KS, et al. Improvement in bronchiectasis on CT imaging in a pediatric patient with cystic fibrosis on ivacaftor therapy. Respiration 2014; 88: 345.

43 Yap VL, Metersky ML. Reversible bronchiectasis in an adult: a case report. J Bronchology Interv Pulmonol 2012; 19: 336-337.

44 Eastham KM, Fall AJ, Mitchell L, et al. The need to redefine non-cystic fibrosis bronchiectasis in childhood. Thorax 2004; 59: 324-327.

45 Cukier A, Stelmach R, Kavakama JI, et al. Persistent asthma in adults: comparison of high resolution computed tomography of the lungs after one year of follow-up. Rev Hosp Clin Fac Med Sao Paulo 2001; 56: 63-68.

46 Piccione JC, McPhail GL, Fenchel MC, et al. Bronchiectasis in chronic pulmonary aspiration: risk factors and clinical implications. Pediatr Pulmonol 2012; 47: 447-452.

47 Gaillard EA, Carty H, Heaf D, et al. Reversible bronchial dilatation in children: comparison of serial high-resolution computer tomography scans of the lungs. Eur J Radiol 2003; 47: 215-220.

48 Liszewski MC, Ciet P, Lee EY. MR imaging of lungs and airways in children: past and present. Magn Reson Imaging Clin N Am 2019; 27: 201-225.

49 Triphan SMF, Biederer J, Burmester K, et al. Design and application of an MR reference phantom for multicentre lung imaging trials. PLoS One 2018; 13: e0199148.

50 Wielputz MO, von Stackelberg O, Stahl M, et al. Multicentre standardisation of chest MRI as radiation-free outcome measure of lung disease in young children with cystic fibrosis. J Cyst Fibros 2018; 17: 518-527.

51 Nguyen AH, Perez-Rovira A, Wielopolski PA, et al. Technical challenges of quantitative chest MRI data analysis in a large cohort pediatric study. Eur Radiol 2019; 29: 2770-2782.

52 Dournes G, Grodzki D, Macey J, et al. Quiet submillimeter MR imaging of the lung is feasible with a PETRA sequence at 1.5 T. Radiology 2016; 279: 328

53 Gibiino F, Sacolick L, Menini A, et al. Free-breathing, zero-TE MR lung imaging. MAGMA 2015; 28: 207-215.

54 Ciet P, Tiddens HA, Wielopolski PA, et al. Magnetic resonance imaging in children: common problems and possible solutions for lung and airways imaging. Pediatr Radiol 2015; 45: 1901-1915. 
55 Scholz O, Denecke T, Bottcher J, et al. MRI of cystic fibrosis lung manifestations: sequence evaluation and clinical outcome analysis. Clin Radiol 2017; 72: 754-763.

56 Woodhouse N, Wild JM, van Beek EJ, et al. Assessment of hyperpolarized 3He lung MRI for regional evaluation of interventional therapy: a pilot study in pediatric cystic fibrosis. J Magn Reson Imaging 2009; 30: 981-988.

57 Ciet $\mathrm{P}$, Bertolo S, Ros M, et al. Detection and monitoring of lung inflammation in cystic fibrosis during respiratory tract exacerbation using diffusion-weighted magnetic resonance imaging. Eur Respir J 2017; 50: 1601437.

58 Tiddens HA, Stick SM, Wild JM, et al. Respiratory tract exacerbations revisited: ventilation, inflammation, perfusion, and structure (VIPS) monitoring to redefine treatment. Pediatr Pulmonol 2015; 50: Suppl. 40, S57-S65. 